\title{
ESTIMATING DAMPING PARAMETERS IN MULTI-DEGREE-OF-FREEDOM VIBRATION SYSTEMS BY BALANCING ENERGY ${ }^{0}$
}

\author{
B. F. Feeny \\ Department of Mechanical Engineering \\ Michigan State University \\ East Lansing, MI 48824 \\ feeny@egr.msu.edu
}

\begin{abstract}
A method of estimating damping parameters for multidegree-of-freedom vibration systems is outlined, involving a balance of dissipated and supplied energies over a cycle of periodic vibration. The power is formulated as the inner product between velocity and force terms, and integrated over a cycle. Conservative terms (mass and stiffness) drop out of the formulation. The displacement response and the input are measured, and the damping coefficients are estimated without knowledge of the mass and stiffness, which can be nonlinear, as illustrated in one example. The identification equations are also obtained with a modal reduction based on proper orthogonal decomposition. The method can be applied with a harmonic motion assumption, or by simple numerical integration. The method is illustrated with linear and quadratic damping, in simulations of a four degree-of-freedom system, and in a string with and without noise.
\end{abstract}

\section{Introduction}

Accurate identification of macroscopic friction models is important to design, analysis and control of vehicles, machines, machine-tool processes, and structures [1], as responses (e.g. friction-induced noise and chatter) are very sensitive to the macroscopic damping model [2-7]. Stability criteria, for example in aerodynamic flutter, are strongly dependent on the system damping properties [8]. Identifying damping can be done via damping-force measurement or damping coefficient estimation.

\footnotetext{
${ }^{0}$ Journal of Vibration and Acoustics, 131 (4) 041005, 2009
}

Measurement of damping forces, such as dry friction, is nontrivial [9], but can be done "directly" with the installation of load cells and the measurement of the contact displacement and velocity (a few examples are [10-12]). "Indirect" damping force measurement [13] involves the measurement of displacements, velocities and accelerations, and balancing of the differential equations of motion to solve for the damping forces.

Friction parameter estimation can be based on general parametric identification schemes [14] applied to measured input and output responses to estimate all unknown parameters. On the other hand, damping parameters can be specifically estimated by making use of vibration properties.

For free vibration, there is the logarithmic decrement for viscous damping $[15,16]$, and the constant decrement for Coulomb damping [17], and combined damping [18-20].

Free vibration decrements are not applicable if the damping is strong enough to thwart sufficient oscillations. For forced vibration, linear viscous friction is estimated from the quality of resonance [21], or the complex motility response [22]. These techniques can produce modal damping in multidegree-of-freedom (MDOF) systems. Recent methods for forced nonlinear oscillators were done in the phase space $[23,24]$. For the case of Coulomb damping only, the resonance cannot be used, as the predicted resonant response is unbounded.

A variety of methods have been proposed for estimating parameters of combined damping sources [25-34]. Energy dissipation was recently applied to estimate the structural loss factor and Coulomb parameter in brush seals for turbomachinery [35]. Tomlinson and Hibbert [36] used power dissipation to estimate Coulomb and hysteretic damping coefficients, and
VIB-07-1094, Feeny 
Liang and Feeny [37] proposed a damping estimation method based on the balance of energy. The idea is to compute the energy input per cycle from input/output measurements, and equate it with the formulated dissipated energy of the damping model to obtain estimation equations for unknown parameter values. The identification equations were consistent with those derived from analytical solutions for the Coulomb-plusviscous case [32]. However the power of the energy balance method lies in the potential application to systems for which analytical solutions are complicated or even unavailable, such as strongly nonlinear systems and MDOF systems. Furthermore, since conservative terms do not dissipate energy, the method can be conducted without knowledge of the mass and stiffness, or even the form of the stiffness, which is free to be nonlinear. The principle should also extend to chaotic responses, analogously to the extension of harmonic balance parameter identification to chaotic responses [38], as the chaos "visits" unstable periodic orbits.

The goal in this paper is to propose an extension of the application of this parametric damping identification idea to MDOF systems with periodic excitation and response. Tomlinson and Hibbert [36] applied their energy balance method to linear MDOF systems with known linear modes, but in the context of determining the equivalent viscous damping. Here, we address more general systems, which can include nonlinear stiffness, and estimate linear and nonlinear damping parameters. Furthermore, modal reduction concepts are united with the energy balance to isolate damping parameters.

\section{Energy Balancing for MDOF Systems}

Multi-body holonomic system equations of motion can be formulated from Lagrange's equations. At a stage of the derivation of Lagrange's equations, we have

$$
\delta \mathbf{q}^{T}\left[\frac{d}{d t}\left(\frac{\partial T}{\partial \dot{\mathbf{q}}}\right)-\frac{\partial T}{\partial \mathbf{q}}+\frac{\partial V}{\partial \mathbf{q}}-\mathbf{Q}_{n c}\right]=0
$$

where, $T$ and $V$ are the kinetic and potential energies, $\mathbf{q}$ is a vector of generalized displacements, and $\mathbf{Q}_{n c}$ includes the nonconservative forces. We separate the nonconservative forces into dissipative and applied forces, i.e. $-\mathbf{Q}_{n c}=\mathbf{Q}_{d}-\mathbf{Q}_{a}(t)$. Then, if the system is scleronomous, upon integration of equation (1) over one cycle of a periodic response, the conservative terms drop out, and we are left with

$$
W_{d}=\oint d \mathbf{q}^{T} \mathbf{Q}_{d}=\oint d \mathbf{q}^{T} \mathbf{Q}_{a}=W_{a}
$$

where $W_{d}$ represents the work of the dissipation forces and $W_{a}$ is the work of the applied forces over one cycle of periodic response. Recasting the integration variable from a displacement differential to a time differential, the identification equation is

$$
\oint \dot{\mathbf{q}}^{T} \mathbf{Q}_{d} d t=\oint \dot{\mathbf{q}}^{T} \mathbf{Q}_{a} d t
$$

To make use of equations (2), the applied force $\mathbf{Q}_{a}(t)$ and responses $\mathbf{q}$ should be measured. The dissipative force vector $\mathbf{Q}_{d}$ is formulated from the dissipation model, chosen by the analyst to be linear in the $p$ unknown damping coefficients, that is in the form of $p$ damping coefficients times respective functions of displacements and velocities. Hence at least $p$ input-output measurement sets are needed.

\subsection{Linear Structures with Nonlinear Damping}

While the principles of equations (1)-(2) are straight forward, implementation can get complicated. Thus we focus our discussion on structural vibrations of the form

$$
\hat{\mathbf{M}} \ddot{\mathbf{x}}+\hat{\mathbf{C}} \dot{\mathbf{x}}+\hat{\mathbf{K}} \mathbf{x}+\hat{\mathbf{f}}(\dot{\mathbf{x}}, \mathbf{x})=\hat{\mathbf{r}}(t)
$$

where $\mathbf{x}$ is the displacement vector and $\hat{\mathbf{r}}(t)$ is the input vector. The identification equations are formed by the inner product of the velocity vector with the equation of motion. Integrating over a period of oscillation, the conservative terms drop out, and the nonconservative work terms are

$$
\begin{array}{r}
W_{d}=\oint d \mathbf{q}^{T} \mathbf{Q}_{d}=\oint \dot{\mathbf{x}}^{T} \hat{\mathbf{C}} \dot{\mathbf{x}} d t+\oint \dot{\mathbf{x}}^{T} \hat{\mathbf{f}}(\dot{\mathbf{x}}) d t, \\
W_{a}=\oint d \mathbf{q}^{T} \mathbf{Q}_{a}=\oint \dot{\mathbf{x}}^{T} \hat{\mathbf{r}}(t) d t .
\end{array}
$$

\subsection{Four-Mass Example}

For illustration, we consider a chain of four equal masses connected by equal linear springs, and linked to the ground on both sides, with linear and nonlinear damping, such that $\mathbf{M}=\mathbf{I}$ is the (dimensionless) mass matrix, and the stiffness, nonlinear damping, and excitation are

$\hat{\mathbf{K}}=\left[\begin{array}{rrrr}2 & -1 & 0 & 0 \\ -1 & 2 & -1 & 0 \\ 0 & -1 & 2 & -1 \\ 0 & 0 & -1 & 2\end{array}\right], \hat{\mathbf{f}}(\dot{\mathbf{x}})=d\left[\begin{array}{c}\dot{\mathbf{x}}_{1}\left|\dot{\mathbf{x}}_{1}\right| \\ \dot{\mathbf{x}}_{2}\left|\dot{\mathbf{x}}_{2}\right| \\ \dot{\mathbf{x}}_{3}\left|\dot{\mathbf{x}}_{3}\right| \\ \dot{\mathbf{x}}_{4}\left|\dot{\mathbf{x}}_{4}\right|\end{array}\right], \hat{\mathbf{r}}(t)=\left[\begin{array}{c}0 \\ 0 \\ 0 \\ a \cos \omega t\end{array}\right]$

The linear damping is proportional to stiffness such that $\hat{\mathbf{C}}=c \hat{\mathbf{K}}, c$ unknown, and the uniform quadratic damping has an unknown constant $d$. The positions are to be measured during a periodically excited periodic response, for four different excitation levels $a$. The number of excitation levels was arbitrarily chosen with the aim of exceeding the number of parameters (two) to be identified, with the thought that redundancy 


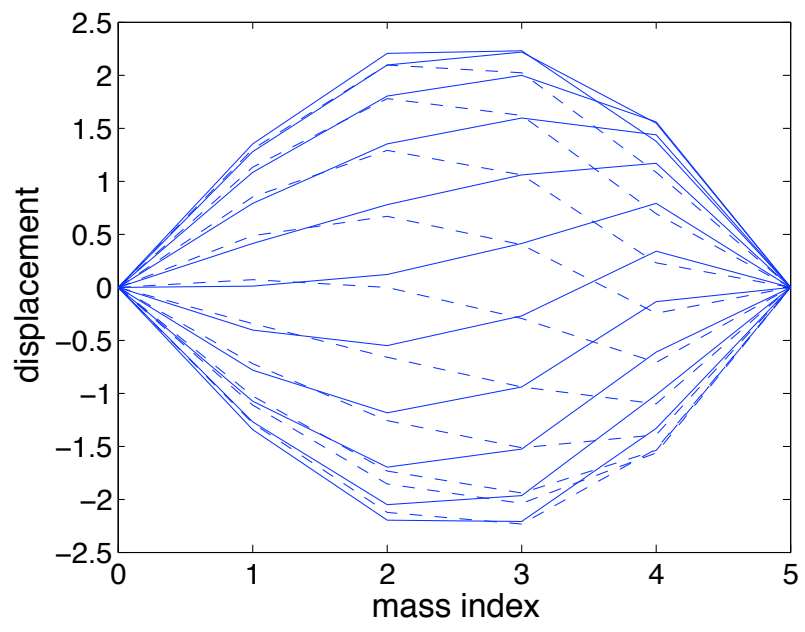

Figure 1. Animated steady state vibration of the four mass system, with nondimensional $a=1$, and with nondimensional axial displacements plotted transversally. The mass deflections are connected with lines for visualization of the instantaneous configurations. The masses are indexed with labels 1 through 4 (mass locations). Mass label 5 indicates the right wall. Solid and dashed lines distinguish forward (upward) and return strokes.

generally helps the least squares fit. (With the model matching the data, little if any, redundancy is needed.)

We simulated responses with the values $c=0.15$ and $d=$ 0.10 . The integration step size was $h=0.1$. The simulations were run for 1000 steps to remove the transients, and then for an additional 200 steps to record steady state responses.

In this example, we resonated the first mode $(\omega=0.618)$ with input amplitudes $a=0.25, a=0.5, a=0.75$, and $a=$ 1. Figure 1 shows the animated displacement of the masses through a cycle of vibration for excitation amplitude $a=1.0$. Figure 2 shows the displacement of mass 4 against the displacement of mass 1 . The loop in the plot indicates asynchronicity, due to the nonlinear damping and the asymmetrically applied excitation (on the forth mass). The effect is only very slightly less pronounced with the lower excitation levels.

Nonetheless, applying a synchronous harmonic response approximation, that is $\mathbf{x}=\left[x_{1}, x_{2}, x_{3}, x_{4}\right]^{T}$ with $x_{i}=X_{i} \cos (\omega t-$ $\phi)$, where $\phi$ is the mean phase lag of the masses with respect to the excitation (a simple approximation motivated by the steady state response of a linear system, but neglecting the variations in phases, apparent in Fig. 2, caused by subdominant modes and damping), we can evaluate the above integrals and express the work terms, such that balancing $W_{d}=W_{a}$ results in

$$
2 \pi \omega c A_{c}+\frac{8}{3} \omega^{2} d A_{d}=\pi a X_{4} \sin \phi
$$

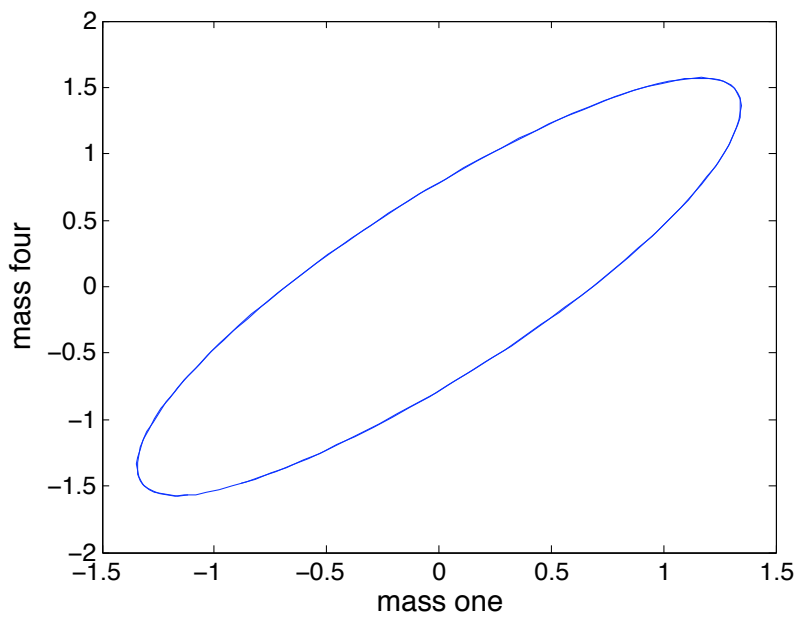

Figure 2. Displacements of mass 4 versus mass 1 show the asynchronicity of the steady state response. The loop in the plot indicates asynchronicity.

where $A_{c}=X_{1}^{2}+X_{2}^{2}+X_{3}^{2}+X_{4}^{2}-X_{1} X_{2}-X_{2} X_{3}-X_{3} X_{4}$, and $A_{d}=X_{1}^{3}+X_{2}^{3}+X_{3}^{3}+X_{4}^{3}$.

The steady state response amplitudes and phases were applied to equation (4), for which the least squares solution produced $c_{h}=0.1341$ and $d_{h}=0.1216$, which are in error by $11 \%$ and $22 \%$. The main source of error is in the synchronicity assumption.

We repeated the parameter estimation without any harmonic approximation. To this end, we express the balance $W_{d}=W_{a}$ as

$$
\oint \dot{\mathbf{x}}^{T} \hat{\mathbf{C}} \dot{\mathbf{x}} d t+\oint \dot{\mathbf{x}}^{T} \hat{\mathbf{f}}(\dot{\mathbf{x}}) d t=\oint \dot{\mathbf{x}}^{T} \hat{\mathbf{r}}(t) d t
$$

Equation (5) has embedded unknowns $c$ and $d$, and has the form

$$
c \alpha+d \beta=\gamma
$$

where, for this particular case of $\hat{\mathbf{C}}$ and $\mathbf{f}, \alpha=\oint\left(\dot{x}_{1}^{2}+\dot{x}_{2}^{2}+\right.$ $\left.\dot{x}_{3}^{2}+\dot{x}_{4}^{2}-\dot{x}_{1} \dot{x}_{2}-\dot{x}_{2} \dot{x}_{3}-\dot{x}_{3} \dot{x}_{4}\right) d t, \beta=\oint\left(\left|\dot{x}_{1}\right|^{3}+\left|\dot{x}_{2}\right|^{3}+\left|\dot{x}_{3}\right|^{3}+\right.$ $\left.\left|\dot{x}_{4}\right|^{3}\right) d t$, and $\gamma=\oint \dot{a} x_{4} \cos (\omega t) d t$. These integrals were performed using the rectangular integration rule. With the same four excitation amplitudes, we wrote four equations of the form (6), and the least squares solution led to estimates $c_{I}=$ 0.1499 and $d_{I}=0.1003$, which have negligible errors.

Since the conservative terms drop out of the formulation of the identification equations (2) and (5), we expect the method to work in the presence of conservative nonlinearity. To test this, a nonlinear spring was grounded to the second 
mass, incurring a force of $x_{2}^{3}$. Simulated responses with the same four excitation amplitudes were periodic but not purely harmonic, and the estimated parameters were $c_{I}=0.1496$ and $d_{I}=0.1036$. These improved to $c_{I}=0.1499$ and $d_{I}=0.1009$ when computed over four cycles, probably showing some sensitivity to the incommensurate sampling rate.

If there were more unknown parameters, for example if each dashpot had a different damping coefficient, or if there were more damping mechanisms at work, then more steadystate responses would be needed to produce more instances of (6) (or equation (4)). These additional steady-state responses could be obtained from more amplitudes of excitation, and/or other resonances. Additional data-generated equations (6) increase the redundancy for the least-squares solution. If there is nonlinearity, for example in one of the damping laws, the multiple amplitudes will produce multiple independent responses.

Liang [39] and Liang [40] independently expanded on [37] and used a coordinate-energy balance for estimating damping in MDOF systems. In these methods, the stiffness values were required, but an identification equation was obtained for each degree-of-freedom. The current paper is a follow-up to [41].

\section{Modal Reduction for Larger Systems}

We now combine the energy balance with modal reduction. This may be helpful when the number of sensors is less than the degree of freedom, for example with "large" MDOF structures or continuous systems.

The modal reduction will be based on proper orthogonal decomposition (POD) [42]. Proper orthogonal modes (POMs) that result from POD represent the principle spatial signal energy distributions in the data. The $n$ POMs that contribute to the significant energy (based on a prescribed criterion, such as $99.9 \%$ of the total energy) will be taken to capture the energy dissipation of the system. The modal energy is indicated by the proper orthogonal value (POV), which can also be interpreted as the mean squared modal coordinate value. The high-degreeof-freedom differential equation model will be projected onto the $n$ POMs, and energy input and dissipation will be determined as functions of the measured input and the parameters and response measurements by means of this projection. The use of modes for model reduction and parameter identification has been widely done in other contexts (e.g. [43-49]).

We illustrate the formulation on linear structures, with added nonlinear damping, of the form (3). Suppose the positions are measured during a periodically excited periodic response, and the proper orthogonal modes are determined (from the eigenvectors of $\mathbf{R}=\mathbf{X}^{T} \mathbf{X} / N$, where the rows of $\mathbf{X}$ are the $N$ time samples of $\mathbf{x}$ ). Note that the POMs do not equal the linear normal modes, although they do converge to linear normal modes in some specific cases [50-53]. Letting $\mathbf{U}$ be the proper orthogonal modal matrix, in which the columns are the POMs, we write $\mathbf{x} \approx \mathbf{U y}$, where $\mathbf{y}$ are the proper orthogonal modal coordinates [54]. If the number of modes are chosen to encompass a large percentage of the signal power (for example 99.9\%), the approximate equality is rather good. Substituting into equation (3) and premultiplying by $\mathbf{U}^{T}$ yields

$$
\mathbf{U}^{T} \hat{\mathbf{M}} \mathbf{U} \ddot{\mathbf{y}}+\mathbf{U}^{T} \hat{\mathbf{C}} \mathbf{U} \dot{\mathbf{y}}+\mathbf{U}^{T} \mathbf{K} \hat{\mathbf{U}} \mathbf{y}+\mathbf{U}^{T} \hat{\mathbf{f}}(\mathbf{U} \mathbf{y}, \mathbf{U} \dot{\mathbf{y}}) \approx \mathbf{U}^{T} \hat{\mathbf{r}}(t),
$$

or

$$
\mathbf{M} \ddot{\mathbf{y}}+\mathbf{C} \dot{\mathbf{y}}+\mathbf{K y}+\mathbf{f}(\mathbf{y}, \dot{\mathbf{y}}) \approx \mathbf{r}(t)
$$

Thus, for the energy balance step,

$$
\dot{\mathbf{y}}^{T}(\mathbf{M} \ddot{\mathbf{y}}+\mathbf{C} \dot{\mathbf{y}}+\mathbf{K y}+\mathbf{f}(\mathbf{y}, \dot{\mathbf{y}})) \approx \dot{\mathbf{y}}^{T} \mathbf{r}(t)
$$

This corresponds to the vector form of the more general equation (1), with

$$
-\mathbf{Q}_{n c}=\mathbf{Q}_{d}-\mathbf{Q}_{a}=\mathbf{C} \dot{\mathbf{y}}+\mathbf{f}(\mathbf{y}, \dot{\mathbf{y}})-\mathbf{r}(t) .
$$

Integrating over a cycle of periodic response, assuming for now to have the same period as the driver (adjustments can be made for subharmonic responses), and noting that the conservative part will have no net change in energy, we have

$$
\oint \dot{\mathbf{y}}^{T}(\mathbf{C} \dot{\mathbf{y}}+\mathbf{f}(\mathbf{y}, \dot{\mathbf{y}})) d t \approx \oint \dot{\mathbf{y}}^{T} \mathbf{r}(t) d t
$$

which is the identification equation, to which multiple input/output measurements will be applied.

\subsection{Four-Mass Example}

First we return to simulations of the example in Section 2 , with $c=0.15$ and $d=0.1$, as the results will be directly comparable. We resonated the system at the first mode $(\omega=$ 0.618 ), let the transients die away, and saved four cycles of periodic data, whence we obtained the POMs, and kept a select number of POMs for the proper orthogonal coordinate (POC) approximation. We did this for four different input amplitudes ( $a=1, a=0.75, a=0.5$, and $a=0.25$ ), for which dominant POM of each response contained 96.96\%, 97.54\%, 98.14\%, and $98.80 \%$, respectively, of the total signal energy, while the second most dominant mode contained 3.04\%, 2.46\%, 1.86\%, and $1.20 \%$ of the total signal energy. As the excitation amplitude decreases, the nonlinear damping contribution decreases, 


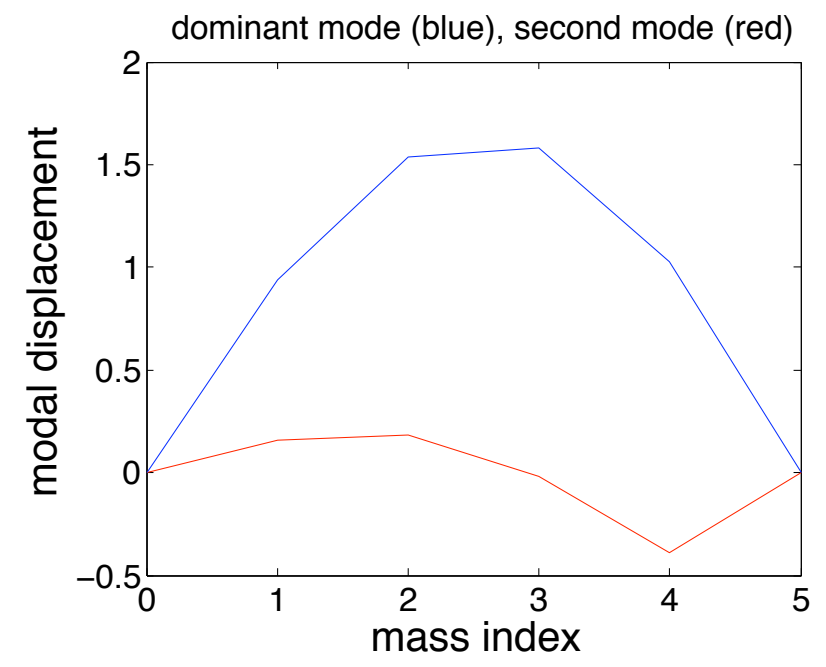

Figure 3. The first two proper orthogonal modes of the four-mass response, to an excitation level $a=1$, normalized to unity and then scaled by the root-mean-squared modal amplitude (square root of the POV) .

and the distortion in the dominant POM from the associated linear normal mode decreases, while the dominant POM becomes increasingly dominant. In all cases, the first two modes together consist of more than $99.99 \%$ of the total signal energy.

For the case of $a=0.25$, the dominant POM is [0.3659, $0.5957,0.6035,0.3834]$, which is sightly unsymmetric due to unsymmetric forcing. The asymmetry in the dominant mode slowly increases with $a$, and for the case of $a=1.0$ the dominant POM is [0.3601, 0.5897, 0.6059, 0.3943]. For the case of $a=1.0$, the dominant and secondary POMs are shown in Figure 3.

Applying a synchronous harmonic approximation with a single POM [41] led to $c_{h}=0.1428$ and $d_{h}=0.1145$, off by about $5 \%$ and $15 \%$.

Using rectangular-rule numerical integrations of the periodic responses, equation (7) takes the form of equation (6), with coefficients

$$
\begin{array}{r}
c \alpha=\oint \dot{\mathbf{y}}^{T} \mathbf{U}^{T} \hat{\mathbf{C}} \mathbf{U} \dot{\mathbf{y}} d t, \quad \beta d=\oint \dot{\mathbf{y}}^{T} \mathbf{U}^{T} \hat{\mathbf{f}}(\mathbf{U} \dot{\mathbf{y}}) d t \\
\gamma=\oint \dot{\mathbf{y}}^{T} \mathbf{U}^{T} \hat{\mathbf{r}}(t) d t
\end{array}
$$

where the integrals are performed by numerical sums.

The term $\beta$ was computed by determining the proper orthogonal modal coordinates $\mathbf{y}$ for each of retained POMs, expressing the original vector $\mathbf{x}$ in terms of the variable $\mathbf{y}$, and then evaluating the nonlinear damping vector $\hat{\mathbf{f}}$ prior to the matrix multiplications in the integrand. When we kept only one POM, the identification results, with the same data, for the numerically integrated coefficients, were $c_{1}=0.1404$ and $d_{1}=0.1138$. Therefore, the quality was very similar to that of the harmonic approximation. Here the error was induced by truncating all but one mode. For nonlinear dynamics signal analyses, we tend to use a stricter percentage threshold for omitting POMs, so this rough approximation is not surprising.

When we kept two POMs, the estimated damping coefficients were $c_{2}=0.1499$ and $d_{2}=0.1003$. In this case, the two leading modes approximately represented the dynamics, and were sufficient to accurately estimate the parameters.

In this basic example, we used "measurements" of all degrees of freedom to obtain the dominant POM, and then applied a reduced number of modal coordinates for the reducedorder parameter estimation, even though the full-order estimation is accessible and was done above. It shows that, if modal information is available, then few modal coordinates may be sufficient in estimating damping parameters. POD provides valuable insight for assessing the number of required modes based on modal energy content. The purpose of this example was to probe the feasibility of reduced-order damping estimation in systems for which it is not possible to measure all of the degrees of freedom, such as a continuous structure, discussed next.

\subsection{Distributed-Parameter Systems}

We stage the problem in terms of the situation for which discrete position measurements are collected from a continuous structure. From these discrete measurements, POMs will be estimated and used as a basis for proper orthogonal modal coordinates (POCs) [54]. We envision projecting the partial differential equation model of the system to a few ordinary differential equations (ODEs) in the POCs. Since the POMs are discrete, the integrals normally associated with a modal projection are discretized by rectangular-rule spatial integration. The conservative terms from the partial differential equation (PDE) are assumed to drop out of the energy balance despite numerical errors that may occur in this discretization, and the damping and input terms are then kept for the energy balance.

As such, the PDE of a damped distributed medium of length $L$ is of the form

$$
\rho \ddot{z}+L_{o} z+c L_{c} \dot{z}+d f(z, \dot{z})=\bar{a}(x, t),
$$

where $z(x, t)$ is the deflection as a function of space $\mathrm{x}$ and time, $\rho$ is the mass per unit length, $L_{o}$ is a conservative linear operator, $L_{c}$ is a linear operator for linear damping, $f$ is a nonlinear damping term, $c$ and $d$ are the damping coefficients for linear and nonlinear damping, and $\bar{a}(x, t)$ is the distributed the periodic excitation. Assuming the system to be sensed at discrete locations $x_{i}, i=1, \ldots, m_{s}$, we obtain a measured displacement 
vector $\mathbf{z}(t)$, with elements $z\left(x_{i}, t\right)$. The ensemble matrix $\mathbf{Z}$ with elements $z\left(x_{i}, t_{j}\right)$ is used for the POD to obtain the $m_{s} \times m$ POM matrix $\mathbf{U}$, where $m$ is the number of retained POMs.

Assuming each $m_{s} \times 1$ POM u to represent a discretized "proper orthogonal modal function" $u(x)$, we can consider a modal projection of equation (10) by the truncated modal expansion $z(x, t) \approx \mathbf{U}(x) \mathbf{y}(t)$, where $\mathbf{U}(x)$ is a $1 \times m$ vector of the $m$ retained "proper orthogonal modal functions" that represent a sufficient portion of the signal energy, and $\mathbf{y}$ is the $m \times 1$ vector of POCs. So the PDE (10) can be written as

$\rho \mathbf{U}(x) \ddot{\mathbf{y}}+L_{o} \mathbf{U}(x) \mathbf{y}+c L_{c} \mathbf{U}(x) \dot{\mathbf{y}}+d f(\mathbf{U}(x) \mathbf{y}, \mathbf{U}(x) \dot{\mathbf{y}}) \approx \bar{a}(x, t)$.

Premultiplying by the $m \times 1$ matrix $\mathbf{U}^{T}(x)$, and integrating over the domain leads to

$$
\begin{array}{r}
\int_{0}^{L} \rho \mathbf{U}^{T}(x) \mathbf{U}(x) d x \ddot{\mathbf{y}}+\int_{0}^{L} \mathbf{U}^{T}(x) L_{o} \mathbf{U}(x) d x \mathbf{y} \\
+c \int_{0}^{L} \mathbf{U}^{T}(x) L_{c} \mathbf{U}(x) d x \dot{\mathbf{y}}+d \int_{0}^{L} \mathbf{U}^{T}(x) f(\mathbf{U}(x) \mathbf{y}, \mathbf{U}(x) \dot{\mathbf{y}}) d x \\
\approx \int_{0}^{L} \mathbf{U}^{T}(x) \bar{a}(x, t) d x
\end{array}
$$

which is an $m \times 1$ vector equation.

For the energy balance, this equation is premultiplied by the transpose of the $m \times 1$ modal velocity vector, and integrated over a period of the response. The conservative kinetic and potential energy terms drop out, leaving the energy balance as

$$
\begin{aligned}
& c \oint \dot{\mathbf{y}}^{T}\left[\int_{0}^{L} \mathbf{U}^{T}(x) L_{c} \mathbf{U}(x) d x\right] \dot{\mathbf{y}} d t \\
& +d \oint \dot{\mathbf{y}}^{T}\left[\int_{0}^{L} \mathbf{U}^{T}(x) f(\mathbf{U}(x) \mathbf{y}, \mathbf{U}(x) \dot{\mathbf{y}}) d x\right] d t \\
& \approx \oint \dot{\mathbf{y}}^{T}\left[\int_{0}^{L} \mathbf{U}^{T}(x) \bar{a}(x, t) d x\right] d t .
\end{aligned}
$$

Since we only have discrete measurements and a discrete POM matrix in place of the continuous $1 \times m$ representation $\mathbf{U}(x)$, the integrals are replaced with spatial sums. Approximating the integrals with rectangular integration, and discretizing the "proper orthogonal modal functions" $\mathbf{U}(x)$ with the $m_{s} \times m$ POM matrix $\mathbf{U}$, we define

$$
\mathbf{A}_{1} \Delta x=\mathbf{U}^{T} \mathbf{L}_{c} \mathbf{U} \Delta x \approx \int_{0}^{L} \mathbf{U}^{T}(x) L_{c} \mathbf{U}(x) d x
$$

For the case of synchronous excitation, $\bar{a}(x, t)=s(x) a(t)$. Discretizing $s(x)$ as an $m_{s} \times 1$ vector $\mathbf{s}$, we define

$$
\mathbf{B} \Delta x=\mathbf{U}^{T} \mathbf{s} \Delta x \approx \int_{0}^{L} \mathbf{U}^{T}(x) d x .
$$

In the above expressions, $\mathbf{L}_{c}$ is the matrix discretization of the linear operator $L_{c}$. (For example, if $L_{c}=\partial / \partial x$, then $\mathbf{L}_{c}$ would be a finite-difference matrix operator, defined to be dimensionally correct.) Also $\Delta x$ is the distance between sampled points, which we will take to be uniform for this discussion. (In the vibrations of two-dimensional media, $\Delta x$ would be replaced by the surface area associated with each measured location.)

The integral $\int_{0}^{L} \mathbf{U}^{T}(x) f(\mathbf{U}(x) \mathbf{y}, \mathbf{U}(x) \dot{\mathbf{y}}) d x$ is more delicate. The nonlinear function is evaluated at each discrete spatial point $x_{i}, i=1, \ldots, m_{s}$, such that $f_{i}=f\left(\mathbf{U}\left(x_{i}\right) \mathbf{y}, \mathbf{U}\left(x_{i}\right) \dot{\mathbf{y}}\right)$. Then a discrete $m_{s} \times 1$ vector $\mathbf{f}$ is built from the elements $f_{i}$. As such

$$
\mathbf{A}_{2} \Delta x=\mathbf{U}^{T} \mathbf{f} \Delta x \approx \int_{0}^{L} \mathbf{U}^{T}(x) \mathbf{f}(\mathbf{U}(x) \mathbf{y}, \mathbf{U}(x) \dot{\mathbf{y}}) d x
$$

Thus $\mathbf{A}_{1}$ is $m \times m$, and $\mathbf{A}_{2}$ and $\mathbf{B}$ are $m \times 1$. Inserting into equation (11) and canceling the $\Delta x$ term yields

$$
c \oint \dot{\mathbf{y}}^{T} \mathbf{A}_{1} \dot{\mathbf{y}} d t+d \oint \dot{\mathbf{y}}^{T} \mathbf{A}_{2} d t \approx \oint \dot{\mathbf{y}}^{T} \mathbf{B} a(t) d t
$$

Finally, the integrals

$$
\alpha=\oint \dot{\mathbf{y}}^{T} \mathbf{A}_{1} \dot{\mathbf{y}} d t, \quad \beta=\oint \dot{\mathbf{y}}^{T} \mathbf{A}_{2} d t, \quad \gamma=\oint \dot{\mathbf{y}}^{T} \mathbf{B} a(t) d t
$$

are numerically evaluated, and the identification equation (15) is

$$
\alpha c+\beta d \approx \gamma
$$

Values $\alpha, \beta$ and $\gamma$ are obtained for each of several periodic steady-state responses, and unknowns $c$ and $d$ are determined by a least-squares solution. The sources of approximation in equation (17) are the spatial discretization limited by the sensor placement, the reduced order modeling based on the dominant POMs, and the numerical integration of the identification equation coefficients, affecting equations (12)-(9).

3.2.1 String Example Strings and cables are common components in real dynamics structures. Here we look at the damped vibrations of a harmonically forced homogeneous string of tension $T$ with linear and quadratic viscous damping. The partial differential equation of motion is

$$
\rho \ddot{z}-T z^{\prime \prime}+c \dot{z}+d \dot{z}|\dot{z}|=\delta\left(x-L_{a}\right) a \cos \omega t,
$$

that is, $L_{o}=-T, L_{c}=1, f(z, \dot{z})=\dot{z}|\dot{z}|$, and $L_{a}$ is the location of the applied excitation. 
The string was simulated by a 4th-order Runge-Kutta procedure on the first ten linear normal modal coordinates. Using normalized modal functions, $\phi_{j}(x)=\sqrt{2 / \rho L} \sin (j \pi x / L)$, of the uniform undamped string, and defining modal coordinates $q_{j}(t)$ such that $z(x, t)=\sum_{j=1}^{\infty} q_{j}(t) \phi_{j}(x)$, the modal coordinates obey

$$
\ddot{q}_{j}+c \dot{q}_{j}+\omega_{j}^{2} q_{j}+d \int_{0}^{L} \phi_{j}(x) f(\dot{z}(x, t)) d x=a \phi_{j}\left(L_{a}\right) \cos (\omega t),
$$

for $j=1,2, \ldots, 10$, where $\omega_{j}=j \pi \sqrt{T / \rho L^{2}}$. In the numerical simulation, the nonlinear damping term in the modalcoordinate ODEs was treated by discretizing the normalized modal functions at eighteen equally spaced points $x_{k}$, evaluating $f(z, \dot{z})=\dot{z}|\dot{z}|$ at the same $x_{k}$, and numerically integrating spatially by the rectangular rule. The parameters were $T=1, \rho=1, L=1, c=0.4, d=0.5$, and $L_{a}=5 / 9$. The undamped modal frequencies were $j \pi, j=1,2, \ldots, 10$. The excitation frequency was $\omega=\pi$, at resonance with the first linear mode. The integration step size was $h=0.1$. The simulation was run for 1000 steps to remove the transients, and then for an additional 200 steps to record steady-state responses.

The modal coordinate data were mapped to physical displacement data via the normalized modal functions. These were sampled at eight evenly spaced locations on the string. In this way we generated the data ensemble $\mathbf{Z}$, as if there were eight displacement sensors on the string. The POD was performed on this ensemble, and the $m$ dominant POMs were retained for matrix $\mathbf{U}$ and the reduced order modeling ( $m=1$ and $m=2$ ). The quantities $\mathbf{A}_{1}, \mathbf{A}_{2}$ and $\mathbf{B}$ were computed according to equations (12)-(14). In particular, from equation (14), $\mathbf{A}_{2}=\mathbf{U}^{T} \mathbf{f}$, where

$$
\mathbf{f}=(\mathbf{U} \dot{\mathbf{y}}) \odot|(\mathbf{U} \dot{\mathbf{y}})|
$$

was used to approximate the discretization of $f(z, \dot{z})=\dot{z}|\dot{z}|$, and where the symbol $\odot$ is meant to represent an element-byelement product between $m \times 1$ vectors resulting in an $m \times 1$ vector, like the ' $*$ ' operation in Matlab.

We applied excitation levels $a=4, a=8, a=16$, and $a$ $=32$. As the amplitude increased, the dominant POM's power share descended from $99.1 \%$ to $95.3 \%$, while the second mode power share ascended from $0.9 \%$ to $4.6 \%$. The total power of the two dominant modes was a minimum of $99.96 \%$ (for the case of $a=32$ ). From this data, the single mode estimation of the damping parameters was $c_{1}=0.3032$ and $d_{1}=0.5354$, and the residuals of equation (17) were negatively biased. The two mode estimation was $c_{2}=0.4024$ and $d_{2}=0.4987$, with unbiased residuals.

Since there is no significant noise or modeling errors, this result shows that error induced by the discretization and

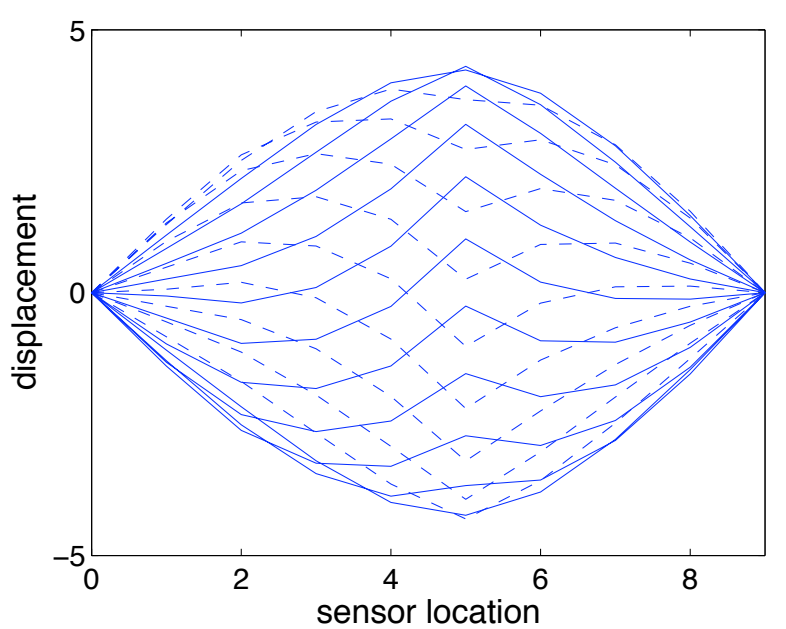

Figure 4. Animated steady state vibration of the nonlinearly damped string, with nondimensional $c=0.4, d=0.5$, and $a=32$. The sensor index numbers indicate the locations of sensed nondimensional displacements on the string. These are plotted and connected with lines to visualize the rest of the string. Sensor label 9 indicates the right wall. Solid and dashed lines distinguish forward (upward) and return strokes.

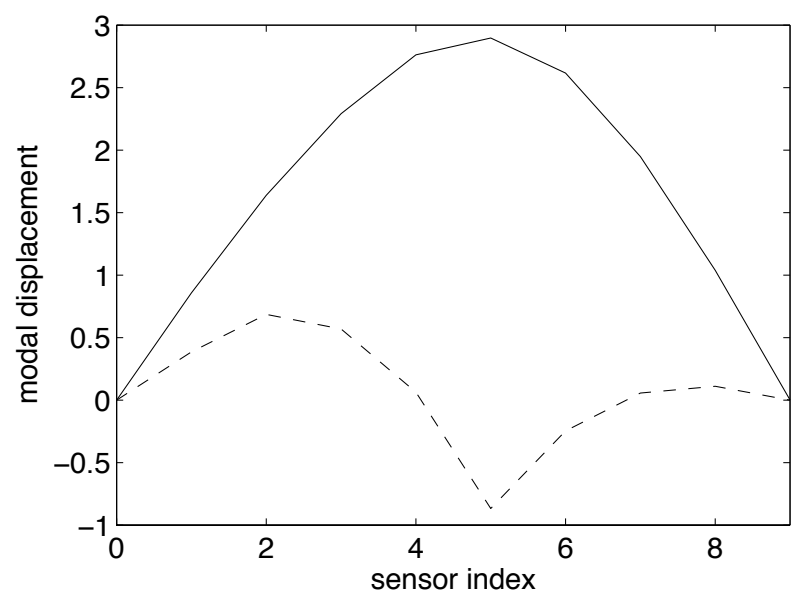

Figure 5. The first two proper orthogonal modes of the string response, normalized to unity and then scaled by the root-mean-squared modal amplitude (square root of the POV).

reduced-order-modeling effects. In the two-mode case, with enough POMs to cover most of the signal energy (more than $99.96 \%$ here), the reduced-order-modeling effect is small.

The animated response is shown in Figure 4, and the first two POMs, scaled by their relative root mean squared modalcoordinate amplitudes, are shown in Figure 5.

Finally, we looked at the effects of sensor noise on the lat- 
ter example. After the numerical integration was completed, and the displacements $z\left(x_{i}, t\right)$ were computed at the simulated sensor sites on the string, a random noise ensemble was added by using the Matlab function 'rand'. This Matlab function produces random numbers uniformly distributed in the unit interval. We scaled these random data to the interval between -1 and 1 , and then by $2^{-3}$. The maximum response amplitude along the string ranged from about 1.3 for the case of $a=4$ to 4.3 for the case of $a=32$. The rms noise value was very close to the theoretical value of $2^{-3} / \sqrt{3}$ for the uniform, zero-mean distribution. The maximum rms noise value represents about $2.4 \%$ of the maximum rms amplitude of the string for the case of $a=32$, and about $7.9 \%$ for the case of $a=4$. The ratio of the maximum noise value to vibration amplitude was larger away from the midpoint.

The noisy displacement ensemble was used to find the dominant POM and its associated POC. The dominant two POMs ranged from $97.82 \%$ and $1.12 \%$ of the signal power, at $a=4$, to $95.21 \%$ and $4.65 \%$, at $a=32$, of the signal energy. The noisy POMs and POCs were applied to the reducedorder identification equations (12) through (17). The resulting estimated damping coefficients were $\hat{c}_{1}=0.1964$ and $\hat{d}_{1}=$ 0.5479 , for the single mode estimation, and $\hat{c}_{2}=0.3935$ and $\hat{d}_{2}=0.5007$, for the two-mode estimation. The one-mode estimation was poorly affected by the noise, but the two-mode estimation error was similar to that of the noise-free estimations. With increased noise levels, once the second POM was lost into the noise level, the results deteriorated to the quality of the single mode approximation.

\subsubsection{Speculation on More Complicated Sys-} tems The examples given here support the concept of the method, and are representative of linear structures with simple nonlinear damping and potentially large degree of freedom. Problems can get more complicated in geometry or in higher dimension. An approach may be to generate a finite-element representation of a 1-D or 2-D distributed system. The finiteelement model will have many more degrees of freedom than sensors. Thus, the system will need to be homogenized to a system with displacement coordinates matching the sensor configuration. The homogenized system will have the form of equation (3). The discrete formulation will carry from there.

If working with a 2-D system with tractable geometry, the approach of Section 3.2 can be adjusted, with modal function matrix $\mathbf{U}(x, y)$, where here $y$ is an additional spatial independent variable. In the discretization of integrals, analogous to equations (12) and (13), the discretization $\mathbf{L}$ of the differential operator, and its compatibility with the modal matrices $\mathbf{U}$, is non trivial. It may be useful to cast these matrices as higher order arrays, and use an appropriate matrix product to produce scalar quantities in the end, or else to discretize into large set of ordinary differential equations (for example by using finite elements) which can then be reduced to form equations (8). In an experimental setting, sensors such as accelerometers, laser transducers [55], or strain gages [56] might be employed, and processed to produce the displacement ensemble.

\section{Conclusion}

We have applied an energy balance method for estimating damping parameters in multi-degree-of-freedom systems and continuous systems with periodic excitation. The method involves an inner product between velocity measurements and force terms in the differential equations. Integrating this inner product over a cycle of periodic response allows the conservative terms to drop out. Hence the identification equations are focused on energy dissipation and excitation terms, and the damping can be estimated without knowledge of the mass and stiffness. Indeed, the method should work for all conservative nonlinear stiffnesses with periodic responses. The approach was supported in numerical examples with linear and nonlinear stiffnesses. A harmonic approximation or a simple rectangular-rule integration can be used. The latter takes less effort and is more accurate.

The method can be combined with a modal reduction scheme. Here, POD was chosen for energy balancing as it produces the optimal and dominant signal energy distributions.

We successfully applied the method to numerical simulations of a four-degree-of-freedom system, which was then compared with the fully sensed MDOF example, and a uniform string. Good results were obtained when the number of modes (two in our examples with asymmetric loading) was sufficient. The identification of the string with random sensor noise was of similar accuracy as the noise-free case. Highfrequency noise should have small effects, as the algorithm involves integration. Low-frequency noise could be more problematic.

The reduced-order examples suggest that the method may be tractable for systems with fewer sensors than the number of physical degrees of freedom. Future tasks will involve the feasibility of the method on experiments, and more complicated systems of more than one dimension.

\section{Acknowledgements}

This material is related to work supported by the National Science Foundation (CMII-0727838). Any opinions, findings, and conclusions or recommendations expressed in this material are those of the author and do not necessarily reflect the views of the National Science Foundation. 


\section{REFERENCES}

[1] R. A. Ibrahim. Friction-induced vibration, chatter, squeal, and chaos: part ii-dynamics and modeling. Applied Mechanics Reviews, 47(7):227-255, 1994.

[2] J. H. Griffin. Friction damping of resonant stresses in gas turbine engine airfoils. Journal of Engineering for Power, 102:329-333, 1980.

[3] J.-H. Wang. Design of a friction damper to control vibration of turbine blades. In F. Pfeiffer A. Guran and K. Popp, editors, Dynamics and Friction: Modeling, Analysis, and Experiment. World Scientific, Singapore, 1996.

[4] Y. Wang. An analytical solution for periodic response of elastic-friction damped systems. Journal of Sound and Vibration, 189(3):299-313, 1996.

[5] J. Guillen and Ch. Pierre. Analysis of the forced response of dry-friction damped structural systems using and efficient hybrid frequency-time method. In Nonlinear Dynamics and Controls, volume ASME DE-Vol. 91, pages 41-50, 1996.

[6] K. Y. Sanliturk and D. J. Ewins. Modeling twodimensional friction contact and its application using harmonic balance method. Journal of Sound and Vibration, 193(2):511-524, 1996.

[7] A. A. Ferri and B. S. Heck. Vibration analysis of dry damped turbine blades using singular perturbation theory. Journal of Vibration and Acoustics, 120(2):588-595, 1998.

[8] D. T. Mook. personal communication, 2002.

[9] S. Filippi, A. Akay, A. May, and M. M. Gola. Measurement of tangential contact hysteresis during microslip. Journal of Tribology-Transactions of the ASME, 126(3):482-489, 2004.

[10] R. A. Ibrahim, Madhavan S., S. L. Qiao, and W. K. Chang. Experimental investigation of friction-induced noise in disc brake systems. International Journal of Vehicle Design, 23(3-4):218-240, 2000.

[11] Osetreich M. Hinrichs, N. and K. Popp. On the modeling of friction oscillator. Journal of Sound and Vibration, 216(3):435-459, 1998.

[12] A. Polycarpou and A. Soom. Transitions between sticking and slipping at lubricated line contacts. In R. A. Ibrahim and A. Soom, editors, Friction-Induced Vibration, Chatter, Squeal, and Chaos, ASME Winter Annual Meeting, volume DE-Vol. 49, pages 139-148, Anaheim, CA, November 8-13 1992.

[13] J.-W. Liang and B. F. Feeny. A comparison between direct and indirect friction measurements in a forced oscillator. Journal of Applied Mechanics, 65(3):783-786., 1998.

[14] J. Beck and K. Arnold. Parameter Identification in Engineering and Science. John Wiley and Sons, New York,
1977.

[15] H. L. F. Helmholtz. On the Sensations of Tone as Physiological Basis for the Theory of Music. Dover, New York, 1877.

[16] Lord Rayleigh. The Theory of Sound, Vol. 1. Dover, New York, 1896. reprinted in 1945.

[17] H. Lorenz. Lehrbuch der Technischen Physik. Erster Band: Technische Mechanik Starrer Gebilde. Verlag von Julius Springer, Berlin, 1924.

[18] A. Watari. Kikai-rikigaku. Kyouritsu (publisher), Tokyo, 1969.

[19] B. F. Feeny and J. W. Liang. A decrement method for the simultaneous estimation of coulomb and viscous friction. Journal of Sound and Vibration, 195(1):149-154., 1996.

[20] J.-W. Liang and B. F. Feeny. Identifying coulomb and viscous friction from free-vibration decrements. Nonlinear Dynamics, 16:337-347, 1998.

[21] F. Förster. Ein neues Messverfahren zur Bestimmung des Elazitätsmoduls und der Dämpfung. Zeitschrift für Metallkunde, 29:109-115, 1937.

[22] D. J. Ewins. Modal Testing: Theory and Practice. Research Studies Press, Letchworth, UK., 1984.

[23] J. P. Cusumano and B. Kimble. Nonlinearity and Chaos in Engineering Dynamics, chapter Experimental observation of basins of attraction and homoclinic bifurcation in a magneto-mechanical oscillator, pages 71-89. John Wiley and Sons, Chichester, 1994.

[24] J. M. Nichols, L. N. Virgin, and H. P. Gavin. Damping estimates from experimental nonlinear time series. Journal of Sound and Vibration, 246(5):815-827, 2001.

[25] R. Stanway, J. L. Sproston, and N. G. Stevens. A note on parameter estimation in nonlinear vibrating systems. Procedings of the Institution of Mechanical Engineers, 199(C1):79-84, 1985.

[26] G. Z. Yao, G. Meng, and T. Fang. Parameter estimation and damping performance of electro-rheological dampers. Journal of Sound and Vibration, 204(4):575584, 1997.

[27] G. R. Tomlinson. An analysis of the distortion effects of coulomb damping on the vector plots of lightly damped system. Journal of Sound and Vibration, 71(3):443-451, 1980.

[28] Q. Chen and G. R. Tomlinson. Parametric identification of systems with dry friction and nonlinear stiffness using a time series model. Journal of Vibration and Acoustics, 118:252-263, 1996.

[29] Duval L. Iourtchenko, D. V. and M. F. Dimentberg. The damping identification for certain sdof systems. In Proceedings of the SECTAM-XX, Developments in Theoretical and Applied Mechanics, pages 535-538, April 16-18 2000. Callaway Gardens, Pine Mountain, GA.

[30] M. F. Dimentberg. Determination of nonlinear damp- 
ing function from forced vibration test of a sdof system. Mekhanika Tverdogo Tela, N. 2:32-34, 1968. (in Russian).

[31] D. V. Iourtchenko and M. F. Dimentberg. In-service identification of nonlinear damping from measured random vibration. Journal of Sound and Vibration, 255(3):549554, 2002.

[32] J.-W. Liang and B. F. Feeny. Identifying coulomb and viscous damping in forced dual-damped oscillators. Journal of Vibration and Acoustics, 126(1):118125, 2004.

[33] M. R. Hajj, J. Fung, A. H. Nayfeh, and S. O. Fahey. Damping identification using perturbation techniques and higher-order spectra. Nonlinear Dynamics, 23(2):189-203, 2000.

[34] H. Liu, B. Liu, D. Yuan, and J. Rao. Identification for sucker-rod pumping system's damping coefficients based on chain code method of pattern recognition. Journal of Vibration and Acoustics, 129(4):434-440, 2007.

[35] A. Delgado and L. San Andrés. Identification of structural stiffness and damping coefficients of a shoed-brush seal. Journal of Vibration and Acoustics, 129(5):648$655,2007$.

[36] G. R. Tomlinson and J. H. Hibbert. Identification of the dynamic characteristics of a structure with coulomb friction. Journal of Sound and Vibration, 64(2):233-242, 1979.

[37] J.-W. Liang and B. F. Feeny. Balancing energy to estimate damping parameters in forced oscillators. Journal of Sound and Vibration, 295(3-5):988-998, 2006.

[38] C. M. Yuan and B. F. Feeny. Parametric identification of chaotic systems. Journal of Vibration and Control, 4(4):405-426, 1998.

[39] J.-W. Liang. Damping estimation via energy-dissipation method. Journal of Sound and Vibration, 307(1-2):949364, 2007.

[40] Y. Liang. Parametric Identification of Chaotic/Nonlinear Systems and Reduced Order Models based on Proper Orthogonal Decomposition. $\mathrm{PhD}$ thesis, Michigan State University, East Lansing, 2005.

[41] B. F. Feeny. Estimating damping parameters in multidegree-of-freedom vibration systems by balancing energy. In Proceedings of the 2007 ASME International Design Engineering Technical Conferences, pages DVDROM paper VIB-35340, Las Vegas, September 4-7 2007.

[42] John L. Lumley. Stochastic Tools in Turbulence. Academic Press, New York, 1970.

[43] Philip M. FitzSimons and Chunlei Rui. Determining low dimensional models of distributed systems. Advances in Robust and Nonlinear Control Systems, ASME-DSC53:9-15, 1993.
[44] M. Spottswood and R. J. Allemang. Identification of nonlinear parameters for reduced order models. Journal of Sound and Vibration, 295(1-2):226-245, 2006.

[45] M. Spottswood and R. J. Allemang. On the investigation of some parameter identification and experimental modal filtering issues for nonlinear reduced order models. Experimental Mechanics, 43(4):511-521, 2007.

[46] M. Amabili, A. Sarkar, and M. P. Päidoussis. Reducedorder models for nonlinear vibrations of cylindrical shells via the proper orthogonal decomposition method. Journal of Fluids and Structures, 18(2):227-250, 2003.

[47] V. Lenaerts, G. Kerschen, and J. C. Golinval. Identification of a continuous structure with a geometrical nonlinearity. part ii: Proper orthogonal decomposition. Journal of Sound and Vibration, 262(4):907-919, 2003.

[48] V. Lenaerts, G. Kerschen, and J. C. Golinval. ECL benchmark: Application of the proper orthogonal decomposition. Mechanical Systems and Signal Processing, 17(1):237-242, 2003.

[49] B. I. Epureanu, L. S. S. Tang, and M. P. Paidoussis. Coherent structures and their influence on the dynamics of aeroelastic panels. International Journal of Non-Linear Mechanics, 39(6):977-991, 2004.

[50] B. Feeny and R. Kappagantu. On the physical interpretation of proper orthogonal modes in vibrations. Journal of Sound and Vibration, 211(4):607-616, 1998.

[51] G. Kerschen and J. C. Golinval. Physical interpretation of the proper orthogonal modes using the singular value decomposition. Journal of Sound and Vibration, 249((5)):849-865., 2002.

[52] B. F. Feeny. On the proper orthogonal modes and normal modes of continuous vibration systems. Journal of Vibration and Acoustics, 124(1):157-160., 2002.

[53] B. F. Feeny and Y. Liang. Interpreting proper orthogonal modes in randomly excited vibration systems. Journal of Sound and Vibration, 265(5):953-966., 2003.

[54] B. F. Feeny. On proper orthogonal coordinates as indicators of modal activity. Journal of Sound and Vibration, 255(5):805-817., 2002.

[55] U. Iemma, L. Morino, and M. Diez. Digital holography and Karhunen-Loève decomposition for the modal analysis of two-dimensional vibrating structures. Journal of Sound and Vibration, 291:107-131, 2006.

[56] M. S. Riaz and B. F. Feeny. Proper orthogonal decomposition of a beam sensed with strain gages. Journal of Vibration and Acoustics, 125((1)):129-131., 2003. 